\title{
Performance Evaluation of Indoor Localization Techniques Based on RF Power Measurements from Active or Passive Devices
}

\author{
Damiano De Luca, ${ }^{1}$ Franco Mazzenga, ${ }^{2}$ Cristiano Monti, ${ }^{2}$ and Marco Vari ${ }^{1}$ \\ ${ }^{1}$ RadioLabs, Consorzio Università Industria-Laboratori di Radiocomunicazioni, Via A. Cavaglieri, 26, \\ 00173 Roma, Italy \\ ${ }^{2}$ Dipartimento di Ingegneria Elettronica, Facoltà di Ingegneria, Università degli Studi di Roma “Tor Vergata," \\ Via del Politecnico 1, 00133 Roma, Italy
}

Received 14 June 2005; Revised 10 May 2006; Accepted 18 May 2006

\begin{abstract}
The performance of networks for indoor localization based on RF power measurements from active or passive devices is evaluated in terms of the accuracy, complexity, and costs. In the active device case, the terminal to be located measures the power transmitted by some devices inside its coverage area. To determine the terminal position in the area, power measurements are then compared with the data stored in an RF map of the area. A network architecture for localization based on passive devices is presented. Its operations are based on the measure of the power retransmitted from local devices interrogated by the terminal and on their identities. Performance of the two schemes is compared in terms of the probability of localization error as a function of the number (density) of active or passive devices. Analysis is carried out through simulation in a typical office-like environment whose propagation characteristics have been characterized experimentally. Considerations obtained in this work can be easily adapted to other scenarios. The procedure used for the analysis is general and can be easily extended to other situations.
\end{abstract}

Copyright () 2006 Hindawi Publishing Corporation. All rights reserved.

\section{INTRODUCTION}

The availability of indoor localization information is helpful to improve existing communication services as well as to create novel and more appealing ones. Several localization techniques have been presented and discussed in the open literature [1-7]. Techniques in [3] focus on the extension of outdoor satellite systems such as the GPS (and in the very near future Galileo) for indoor operations. They use indoor GPS signal repeaters and high-sensitivity receivers for the position calculation of fixed or nomadic devices. Results are very interesting but the real-time tracking of indoor mobile terminals in every location inside the building could be problematic.

Ultra-wideband-(UWB-) based communication networks currently under study $[8,9]$ also offer indoor localization at, practically, no additional costs. In fact, due to the very large bandwidth allocated to UWB signals, position information based on the time difference of arrival can be very accurate also for indoor environments. Even though UWB technology seems to be very promising, UWB-based localization systems and algorithms are still under study [9] and their performance could be compared with the results presented in this paper that focus on other technologies and techniques for indoor localization.

In order to improve existing communication services with localization, it is necessary to integrate the communication and the localization networks at some protocol level. In many cases, this integration is straightforward especially when the existing wireless communication infrastructure can be reused to include the localization feature with minor protocol modifications. An example is given in [4] where the received power of the signals transmitted by the access points (APs) of the wireless local area network (WLAN) is compared with those stored in the RF map of the area to achieve localization. The main techniques for indoor localization based on the measurement of the received power at the terminal provide the simplest and maybe the cheapest approach to include the localization feature inside an existing communication infrastructure. Implementation of positioning methods based on time-delay measurements is generally more complex even though better position accuracy can be achieved provided that (indoor) multipath effects are adequately mitigated.

The generic localization procedure based on RF power measurements can be divided into two phases: 
(1) the terminal measures the received power(s) of the signals transmitted by some devices used for localization;

(2) power samples are processed (somewhere) to estimate the position of the terminal.

The data processing phase can be centralized or distributed. In the first case, power data are retransmitted by the terminal to a local server, while in the second case the terminal owns all the required side information necessary to calculate position. The distributed approach may require the terminal to store and to (continuously) update the side information such as the location of the transmitting fixed points and so forth. This may lead to an unnecessary complexity of the entire system. In many cases, the centralized approach seems to be preferable, see [2]. Several data processing techniques mostly based on RF power measurements at the terminal have been proposed in the literature $[2,6]$. Typical implementations of this techniques adopt (common) communication technologies such as IEEE 802.11 [10], Bluetooth [11], and so forth. In general, the accuracy of position estimation depends on the propagation characteristics of the specific environment, on the number of transmitting devices, and on the resolution of the RF-radio map. In general, it can be observed that the accuracy of localization information greatly suffers for the presence of fading due to obstacles. As an example, in an office with moving persons, doors (closed or opened), the environment characteristics rapidly change and this can lead to a significant departure of the instantaneous powers measured by the terminal from those stored in the radio map. Another relevant factor influencing the position estimate is the power measurement accuracy guaranteed by the hardware inside the terminal to be located. In this paper, we present a statistical characterization of power measurement errors based on experimental data and we show that these inaccuracies may turn out in (severe) localization errors. The performance of these techniques can be slightly improved with the use of motion prediction and estimation based on Viterbilike algorithms or Kalman filters and so forth [6]. However in all cases, the precision of the estimated position is always related to the resolution of the radio map.

Until now, the attention was focused on localization techniques based on active devices transmitting beacon-like signals. In this paper, we present a novel localization system based on passive devices of the RFiD type and we show that very good localization performance can be obtained with respect to the active case. The architecture of the proposed passive localization system is shown in Figure 1. The system comprises several passive devices scattered in the service area. The terminal to be located sends a signal in broadcast to interrogate the passive devices. The power transmitted by the interrogator is selected so that only the passive devices in the close proximity of the terminal respond to the interrogation. The identities of the answering passive devices and, possibly, the corresponding received powers are recorded by the terminal and are sent to a central server that processes the data in order to estimate the position of the terminal. The identities of the devices are used to restrict the area where terminal is located while the information on the measured power can be used to refine the position calculation. With

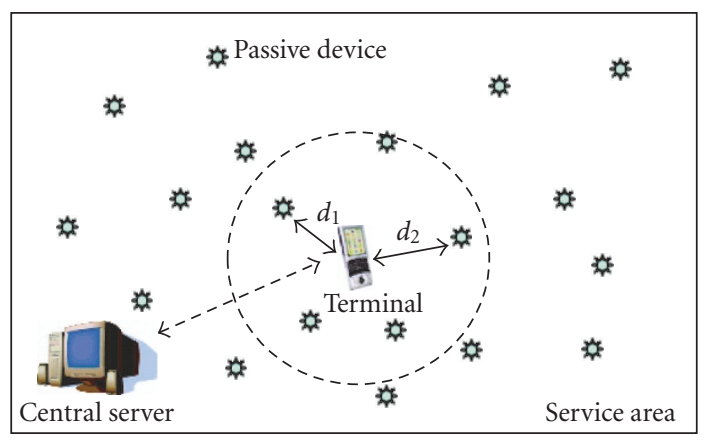

FIGURE 1: Architecture of the considered passive localization system.

high probability, only devices in line of sight (LOS) will respond to the interrogation so that the free-space propagation model can be used to relate the received power level to the distance $d_{i}, i=0,1, \ldots$, (see Figure 1 ) between the terminal and the $i$ th passive device.

The performance of the localization techniques based on passive devices mainly depends on their density that, due to the low cost of passive devices (simple labels), can be very high. Once passive devices have been placed in the area and their positions and identities have been registered to the local central server, no further maintenance of the system is required. ${ }^{1}$ In a passive system, the selection of the multipleaccess strategy required to avoid collision among the signals reaching the terminal is another important aspect for system design. Some techniques have been presented in [12] but it is out of the scope of the paper to discuss them and we assume that collision resolution is ensured. One simple and effective method based on time backoff is briefly described in Section 4.

To analyze the performances of the considered active and passive localization techniques, we introduce the probability of localization error $P_{p}$ as a function of the number/density of devices used for localization. In the active case, we define the $P_{p}$ as the probability that the measured position is outside a circle of radius $1.5 d$, where $d$ is the step of the regular grid of points representing the RF map of the area. The circle is centered in the actual terminal position. In the passive case, the $P_{p}$ is defined as the probability that the distance between the estimated position and the actual position is greater than $1 \mathrm{~m}$. Results on $P_{p}$ are obtained through simulation in a realistic office environment and accounting for power measurement errors due to the hardware characteristics of the terminal. The measurement errors are characterized in terms of an additive Gaussian noise (in $\mathrm{dB}$ ) to be added to the average power value. In order to analyze the performance in the active case, we use an extended multiwall propagation model that was developed on the basis of experimental measurements obtained during a campaign conducted within the University of Rome "Tor Vergata," see [13].

\footnotetext{
${ }^{1}$ Except for the normal routine including the identification and substitution of faulty devices.
} 


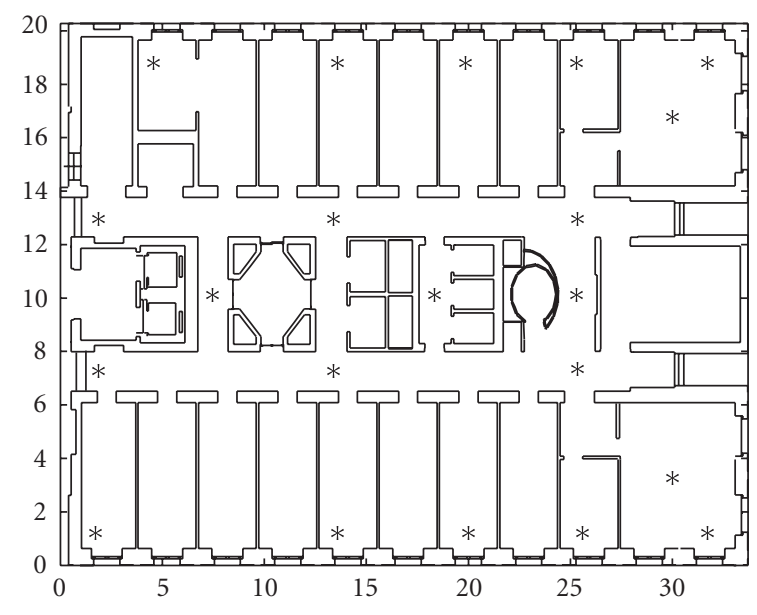

Figure 2: Schematic representation of the considered scenario; the asterisks indicate the position of the active devices; area is $33.7 \times$ $20 \mathrm{~m}^{2}$.

It is shown that with an increase in the number of active devices, a $P_{p}$ decreases, but due to power measurement noise and quantization effects, it cannot be reduced below a limiting value. The dependence of the $P_{p}$ on the density of passive devices in the area is studied. We show that the increase in the number of passive devices can be helpful to reduce the $P_{p}$ even in the presence of power measurement errors. In fact, when the number of passive devices responding to the interrogation is relatively large ( 4 or more), relatively accurate localization can be achieved only using the identities of the responding devices. In fact, this information can be used by the server to determine the terminal uncertainty area defined as the intersection of the coverage areas of the responding devices.

The paper is organized as follows: in Section 2 we illustrate the realistic office-like scenario considered in the paper. In Sections 3 and in 4 we analyze the limiting performance of the active and passive localization techniques, respectively. Finally in Section 5, conclusions are drawn.

\section{SCENARIO DESCRIPTION}

The topology of the considered office scenario is depicted in Figure 2. The environment is characterized by small rooms aligned along two parallel corridors. Offices are accessed through fireproof doors. Small/medium-size walls are dominant in this kind of environment.

\subsection{Multiwall channel model}

Due to the small number of transmitting devices in the area, in general it is not possible to apply simple propagation models, such as free space, to relate the received power to distance. For this reason, we need to consider more complex propagation models accounting for the geometry of the environment.

In this paper we consider the multiwall path loss model presented in [13] which accounts for propagation at $2.4 \mathrm{GHz}$. It was obtained by the authors during an experimental
TABLE 1: List and the meaning of the multiwall model parameters.

\begin{tabular}{|c|c|}
\hline$M_{w}$ model parameter & Meaning \\
\hline$l_{c}=47.4$ & Constant factor $(\mathrm{dB})$ \\
\hline$l_{1}=3.8$ & $\begin{array}{l}\text { Attenuation due to light wall }(\mathrm{dB}) \text { : } \\
\text { thickness }(0,20] \mathrm{cm}\end{array}$ \\
\hline$l_{2}=3.9$ & $\begin{array}{l}\text { Attenuation due to medium wall }(\mathrm{dB}) \text { : } \\
\text { thickness }(20,40] \mathrm{cm}\end{array}$ \\
\hline$l_{3}=5.7$ & $\begin{array}{l}\text { Attenuation due to heavy wall }(\mathrm{dB}) \text { : } \\
\text { thickness }(40,60] \mathrm{cm}\end{array}$ \\
\hline$l_{4}=12.4$ & $\begin{array}{l}\text { Attenuation due to external building } \\
\text { wall }(\mathrm{dB}) \text { : thickness }(60,80] \mathrm{cm}\end{array}$ \\
\hline$l_{d}=1.4$ & Attenuation due to normal door (dB) \\
\hline$l_{f d}=10.2$ & Attenuation due to fireproof door $(\mathrm{dB})$ \\
\hline $10 \gamma=23.2$ & Propagation exponent \\
\hline
\end{tabular}

campaign within the office buildings of the University of Rome "Tor Vergata." It is based on generalization of the classical one slope loss model including an additional attenuation term due to losses introduced by the walls and floors encountered by the direct path between the transmitter and the receiver, that is,

$$
L(d)=L_{O S}(d)+M_{w}(d B)
$$

where $L_{O S}(d)$ is

$$
L_{O S}(d)=10 \gamma \log _{10}(d)+l_{0},(d B)
$$

and $\gamma$ is the path loss exponent, $d$ is the direct transmitterreceiver distance in $m$, and $l_{0}$ is the minimum coupling loss. The $M_{w}$ in (1) is the multiwall component that, for our purposes, is expressed as

$$
M_{w}=l_{c}+\sum_{i=1}^{I} k_{w i} l_{i}+\sum_{n=1}^{N_{d}} \chi_{n} l_{d}+\sum_{n=1}^{N_{f d}} \lambda_{n} l_{f d}(\mathrm{~dB}),
$$

where $l_{c}$ is a constant, $k_{w i}$ is the number of penetrated walls of type $i, l_{i}$ is the attenuation due to the wall of type $i$, $i=1,2, \ldots, I, N_{d}$ and $N_{f d}$ are the numbers of normal and fireproof doors encountered by the direct path, and $\chi_{n}\left(\lambda_{n}\right)$ are binary variables accounting for the status of the $n$th door ( $n$th fireproof door). ${ }^{2}$ The meaning of the parameters in (3) is summarized in Table 1 . The constant $l_{c}$ in (3) includes the constant $l_{0}$ in (2). The main advantages of using a multiwall model lies in its simplicity as compared to other techniques and in the possibility to calculate losses accounting for some physical characteristics of the propagation environment (e.g., the thickness of the walls traversed by the direct electromagnetic path). In addition, our model also includes the (nonnegligible) loss introduced by fireproof doors in accordance

${ }^{2}$ Open: $\chi_{n}=0\left(\lambda_{n}=0\right)$, closed: $\chi_{n}=1\left(\lambda_{n}=1\right)$. 
to their status [13]. Limitations of the multiwall models as compared to more complex ray-tracing techniques have been analyzed in the current literature [14].

\subsection{Characterization of measurement noise}

The understanding of the power measurement errors due to the hardware in the terminal is important especially when the propagation map of the area (see the next section) is created on the basis of experimental data. To obtain power measurements, we used a portable device equipped with a standard IEEE 802.11 adapter. In order to characterize the power measurement accuracy of the adapter, we considered some commercial devices provided by different manufacturers. In Figure 3 we plot the average power measured by fixed adapters receiving from an 802.11 AP under line-of-sight (LOS) propagation conditions. Data have been collected considering different communication channels. From data in Figure 3, it can be observed that adapters by different manufacturers provide different values of the average received power (up to $5 \mathrm{~dB}$ of variation) depending on the selected communication channel. This fact has to be accounted for in the creation of the RF map.

We also investigated the temporal coherence of the power measurements. We fixed the position of the AP and of the adapter in LOS conditions and we sampled the received signal power each two seconds for a time interval of four hours, thus obtaining more than 7000 samples. It was observed that power measurements are quantized and they can significantly fluctuate around their mean. This fact is shown in Figure 4 where we plot the statistics of the received power of the AP beacon for a fixed terminal adapter operating in LOS propagation conditions. Power fluctuations are not negligible and a Gaussian statistics (in $\mathrm{dB}$ ) with standard deviation of $\sigma \cong 2.5 \mathrm{dBm}$ fits well to measurements. Power fluctuations can influence the performance of the localization algorithms based on the RF map which is commonly built using the average power values.

\section{LOCALIZATION BASED ON ACTIVE DEVICES}

The positioning of the active devices in the area is a critical issue for the performance of the localization network. In an IEEE 802.11-based system, the access points (APs) can be positioned to achieve the best coverage, thus reducing the overlap among the coverage areas. Obviously, this could not be optimal for localization where it is necessary to increase the number of APs simultaneously seen by the terminal. In addition, in order to save the costs of the communication network, coverage should be obtained using a suitable planning aiming at minimizing the number of the APs. In this case, the terminal to be located could receive one or two APs at maximum and, as shown in the following, this can impair the performance of the localization algorithm.

To assess the effectiveness of the active network for localization, in the following we evaluate the limiting performance of a localization technique based on the RF map of the area. Performance is expressed in terms of the localization error probability $P_{p}$. The considered technique operates

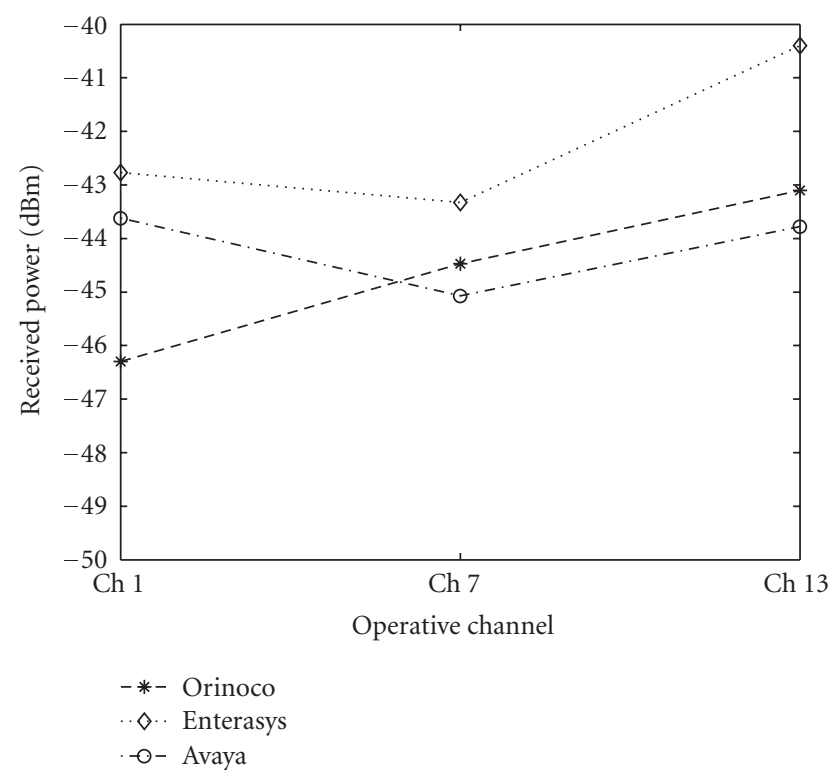

FIGURE 3: Received average power on different WLAN channels; AP transmitter power of $17 \mathrm{dBm}$.

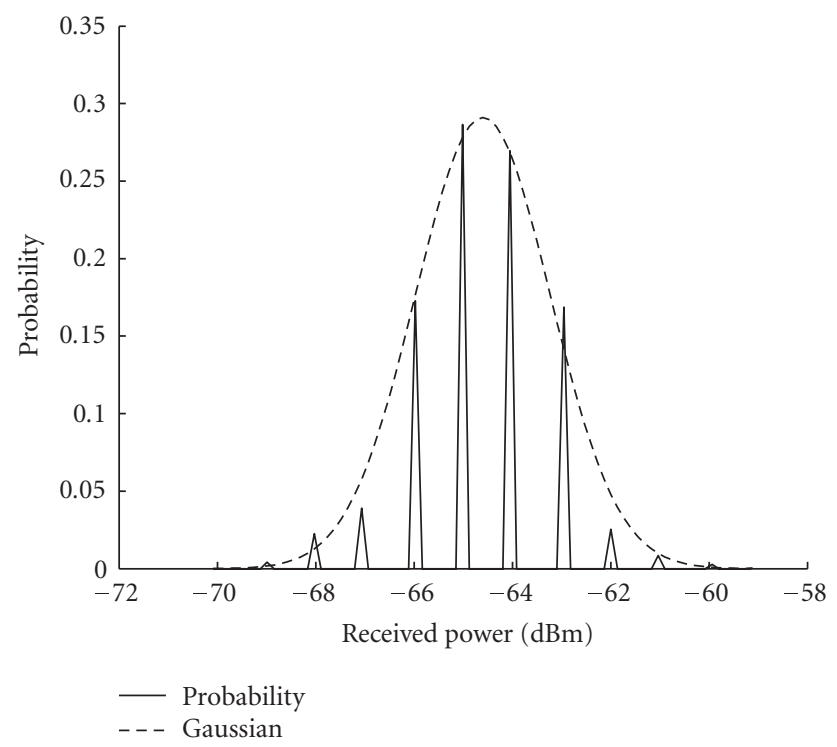

FIGURE 4: Statistics of the average received power for a terminal adapter.

as follows:

(1) the terminal acquires the identity of the transmitting devices inside its coverage area, and for each one it measures the corresponding received RF power;

(2) these data are transmitted to a central server to determine the position of the terminal.

\subsection{Position estimation}

The position estimation algorithm is based on the RF map of the area. The RF map is a database containing the power 
received by each AP for each point $\left(x_{i}, y_{i}\right)$ positioned over a (regular) grid covering the entire service area. The RF map data are organized in an array. Each row refers to the powers received on the single grid point due to the active devices each one indexing one column of the array. The identities of the devices help to select the columns of the RF map to be used in the position calculation. Indicating with $\mathbf{w}=\left(w_{1}, w_{2}, \ldots, w_{n}\right)$ the vector of the measured power, it is compared with the stored power vectors $\mathbf{W}_{i}=\left(W_{i 1}, W_{i 2}, \ldots, W_{i n}\right), i=1, \ldots, N_{\text {points }}$, where $N_{\text {points }}$ is the number of grid points. The $\mathbf{W}_{i}$ contains the powers measured in the $i$ th grid point due to the transmission of the $n$ selected active devices.

The point in the RF map resulting at minimum distance from $\mathbf{w}$ is selected as the position estimate of the terminal. From the work in [6], the Euclidean metric gives better results with respect to the other methods. In this case we assume that the terminal is positioned in the $j$ th point in the RF map grid, that is, $\left(x_{j}, y_{j}\right)$, such that

$$
j=\arg \left\{\min _{i=1, \ldots, N_{\text {points }}}\left\|\mathbf{w}-\mathbf{W}_{i}\right\|^{2}\right\},
$$

where $\|\cdot\|^{2}$ is the quadratic vector norm. From (4), it can be observed that when using the RF map, the minimum resolution in the position estimation is related to the grid step (d). Expanding (4), the minimization problem is equivalent to searching for the index $j$ corresponding to the minimum component of the vector

$$
\mathbf{O}=\mathbf{E}-2 \mathfrak{R}\left\{\mathbf{W}^{H} \mathbf{w}\right\},
$$

where $E=\left[\begin{array}{llll}E_{1} & E_{2} & \cdots & E_{N_{\text {points }}}\end{array}\right]^{T}$ is a vector with components $E_{i}=\mathbf{W}_{i}^{H} \mathbf{W}_{i} ; \mathbf{W}$ is an $n \times N_{\text {points }}$ matrix with columns equal to the RF map grid vectors $\mathbf{W}_{i}, i=1,2, \ldots, N_{\text {points. }}$.

\subsection{Simulation results}

The calculation of $P_{p}$ in a closed analytical form seems to be a very difficult task since it depends on several parameters such as the number of active devices turned on in the area, their positions, the instantaneous propagation conditions (fast fading due to obstacles in the area), the accuracy of the power measurement in the terminal, the accuracy of the RF map, and on the topology of the area.

In order to evaluate the limiting performance of the localization algorithms based on the RF map in terms of $P_{p}$, we considered the following simulation scenario. A maximum number $N_{S}=21$ of active devices have been positioned in the area trying to avoid undesired clusterings. Their layout is shown with the asterisks in Figure 2. The transmission power of the single active device was set to $w_{T}=20 \mathrm{dBm}$. In order to evaluate $P_{p}$ under very general operating conditions, we randomly locate the terminal in the area and for each position we evaluate the vector $\mathbf{w}$ containing the RF powers $w_{i}$ received from the active device inside the coverage area of the terminal. To account for realistic measurements, the received powers calculated with the multiwall model in (1) have been corrected by adding a zero-mean Gaussian error

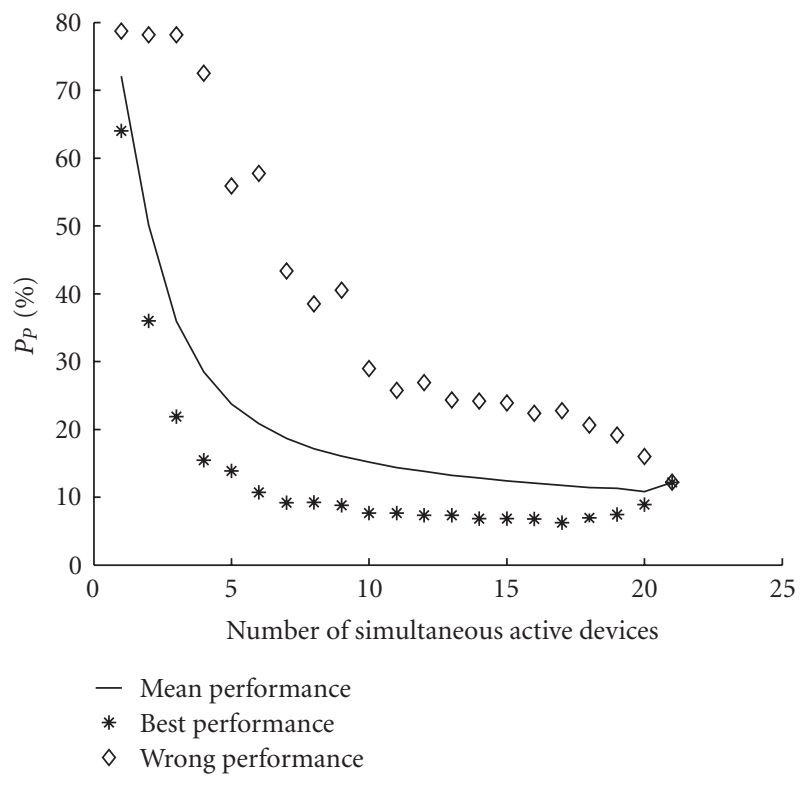

Figure 5: $P_{p}$ as a function of the number of active devices in the area.

with standard deviation $\sigma=2.5 \mathrm{~dB}$ and their values have been quantized with a step of $1 \mathrm{dBm}$. The terminal receiver sensitivity was set to $S=-\infty \mathrm{dBm}$ so that it is able to measure the power coming from every active device in the area. The last assumptions is obviously unrealistic but it is helpful to provide a lower bound on the localization system performance. In order to evaluate the best achievable performance, no fast fading effects were considered. For each terminal location, the position estimate was evaluated in accordance to the algorithm described in the previous section. Several layouts of the active devices have been considered. During simulation, the number of active devices used for localization was varied from 2 up to $N_{S}$. Indicating with $N_{A}$ the number of active devices (ADs) used for localization $\left(N_{A}=2,3, \ldots, N_{S}\right)$, for each $N_{A}$ different layouts of the active devices were considered. They were obtained by randomly switching on and off the $N_{S}$ available devices. Calculations were repeated for several positions of the terminal in the area and considering variable $N_{A}$. For each $N_{A}$, calculation of $P_{p}$ was repeated several times (5000) and considering different layouts. The $P_{p}$ was evaluated as the ratio of the number of times the estimated position was outside $1.5 \mathrm{~d}$ from the actual position of the terminal and the total number of trials.

In Figure 5 we plot the average $P_{p}$ as a function of the available devices in the area. The RF map grid step was set to $d=2 \mathrm{~m}$. In Figure 5 the maximum, the mean, and the minimum values of the average $P_{p}$ have been indicated. The large variations in the $P_{p}$ are due to the geometric arrangement of the active devices used for localization. In particular, since the ADs participating in the localization are randomly selected in each iteration, it was observed that the largest values of $P_{p}$ can be obtained when the ADs used for position measurement result to be located along a straight line and almost LOS conditions exist with the terminal. In this case 
due to the symmetric configuration, the same power vector may indicate different points in the area. Another case corresponding to large values for $P_{p}$ occurs when the ADs are (randomly) concentrated within a relatively small area as compared to the service area. In this case for several points in the area, the differences among the power vectors are not so marked, and due to measurement errors, localization errors can occur. Better performance corresponding to the minimum values of $P_{p}$ in Figure 5 was obtained when no particular symmetries exist in the layout of the ADs and/or when the ADs are sufficiently sparsed in the area. When $N_{A}=N_{S}=21$, the three curves intersect since the layout of the devices is unique. $^{3}$

It is interesting to observe that even increasing the number of active sensors in the area, the $P_{p}$ cannot decrease below a threshold even in the best cases. This is due to noise and quantization error in the power measurements influencing the result of the comparison with the data in the RF map as in (4). Data in the RF map have been obtained from simulation neglecting any noise effect. This assumption is representative of a realistic situation since the measured powers, used to create the RF map, are commonly obtained by averaging them over a long time and using accurate instrumentation. In Figure 6 we plot the mean $P_{p}$ as a function of $N_{A}$ when power measurements are affected by quantization error with and without noise. When only quantization noise is considered, the performance lower bound is obtained.

The dependence of $P_{p}$ on the terminal receiver sensitivity is shown in Figure 7 where we plot the mean $P_{p}$ for two different values of $S$, for example, $S=-90 \mathrm{dBm}$ and $S=-110 \mathrm{dBm}$.

The improvement in the receiver sensitivity allows to increase the number of active devices seen by the terminals, thus providing better localization performance. However when active devices are also used to provide communication services (such as the APs in the IEEE 802.11a,b network), the visibility of more than one active device from the terminal to be located could lead to interference situations that impair the normal operation of random access schemes such as the carrier-sense multiple access with collision avoidance (CSMA/CA).

To analyze the performance of the localization algorithm including memory and tracking of the terminal position, we reimplemented the Viterbi-like technique in [7]. Results on $P_{p}$ as a function of the number of active sensors in the area are reported in Figure 8. To obtain the data in Figure 8, we assumed that terminals moved along some predefined routes in the office area. For each reference point in the route, we evaluated the position with the algorithm in (4) and we compared it with the exactposition of the terminal. From

\footnotetext{
${ }^{3}$ The goodness of one configuration of ADs with respect to another one for localization could be appreciated looking, for example, at the minimum distance among power vectors calculated for the same set of ADs to be used for a terminal when it is located in a specific region of the service area. If the minimum distance among these power vectors is zero or comparable to the power measurement error, localization in that region could be problematic and possibly the ADs should be repositioned.
}

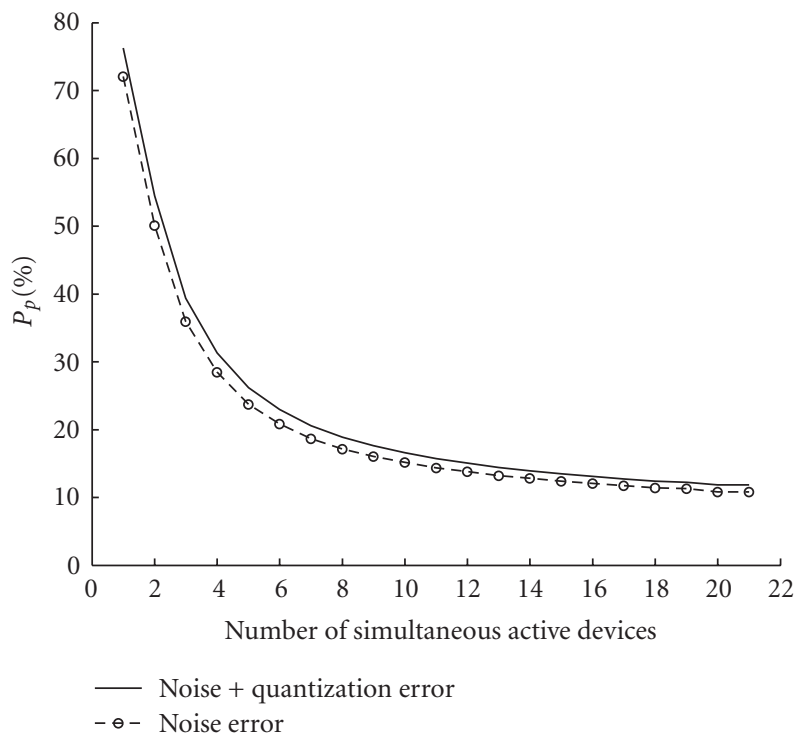

Figure 6: $P_{p}$ as a function of the number of active sensors in the area; noise and quantization error (continuous line); quantization error alone (dashed line).

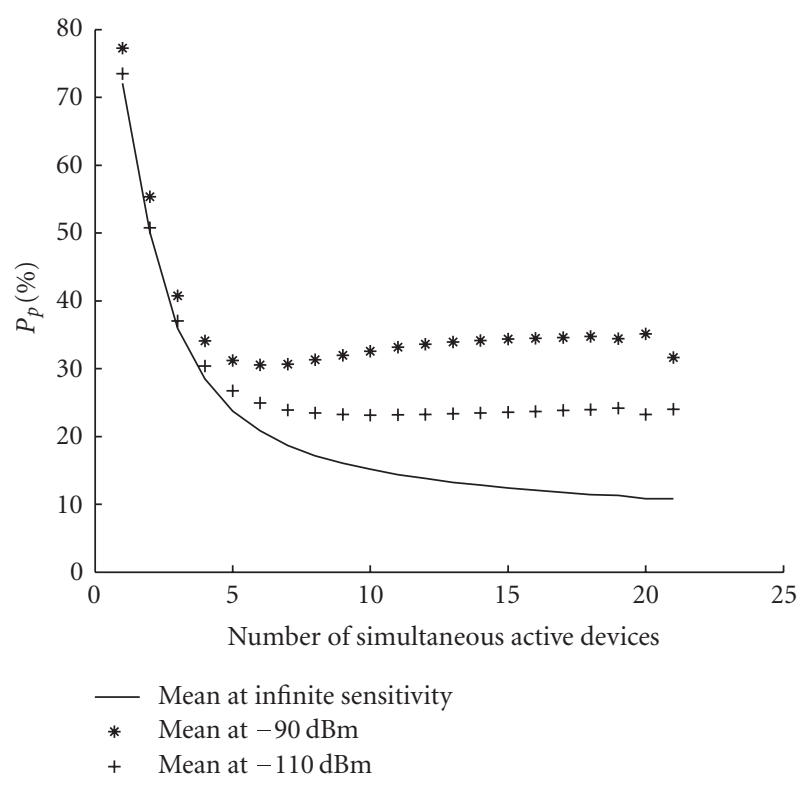

FIgURe 7: $P_{p}$ as a function of the number of active sensors in the area; $S=-90 \mathrm{dBm}$ and $S=-110 \mathrm{dBm}$.

Figure 8 , it can be observed that the improvement due to the addition of the terminal tracking features is modest at the expense of a greater complexity.

\section{LOCALIZATION BASED ON PASSIVE DEVICES}

In this section, we evaluate the performance of the proposed localization network based on passive devices densely scattered in the area shown in Figure 2. The terminal sends an interrogation signal to the neighboring devices that respond to the terminal providing their identities. In the simplest case, 
to avoid interference, the answers coming from passive devices can be delayed by a random backoff time. In case of collision at the terminal receiver, interrogation can be repeated until every response is correctly received. Other, and more complicated, procedures to avoid or reduce collisions are illustrated in [12]. The terminal should also be able to measure the received power $w_{i}$ of the $i$ th responding device that can be related to the device-terminal distance $d_{i}$ by the equation

$$
w_{i}=\frac{w_{T}(\lambda / 4 \pi)^{4} G_{t x}^{2} G_{r x}^{2}}{d_{i}^{4} I_{L}}
$$

where $\lambda=0.125 \mathrm{~m}$ is the wavelength associated to the operating frequency $(2.4 \mathrm{GHz}), I_{L}$ is the passive device insertion loss, $G_{t x}$ and $G_{r x}$ are the transmitting and receiving antenna gains, and $w_{T}(\mathrm{~W})$ is the transmitted power of the interrogating signal.

The central server estimates the terminal position on the basis of the identities of the responding devices and the measured $w_{i}$ (see Figure 1). The identities of the responding devices allow to restrict the area where the terminal is located. They can also be used to determine the uncertainty area obtained as the intersection of the coverage areas of the responding devices. The position estimate within the uncertainty area can be refined using the values of $w_{i}$ in (6). Inverting (6) with respect to $d_{i}$, a position estimate $(x, y)$ of the terminal can be obtained solving the (overdetermined) nonlinear system of equations:

$$
d_{i}^{2}=\left(x-x_{i}\right)^{2}+\left(y-y_{i}\right)^{2}, \quad i=1,2, \ldots, N_{r},
$$

where $\left(x_{i}, y_{i}\right)$ are the coordinates of the $N_{r}$ responding devices. The solution of the system in (7) was obtained using standard algorithms implemented in the fsolve routine of Matlab.

\subsection{Simulation results}

The passive devices used for localization have been positioned as depicted in Figure 9 where the coverage areas of a reduced set of devices have been depicted. The arrangement of RFID devices in Figure 9 is only for illustrative purposes. The total number of RFID devices considered for simulation is higher than that in Figure 9. It is further assumed that devices cannot reradiate through walls. Similarly to the active case, in order to simulate different densities, the number of passive devices participating in the localization was varied during simulation. In particular, we randomly "turned off" some of the devices participating in the localization in accordance to a uniform distribution. ${ }^{4}$ For each one of the selected RFID densities, we repeated the turning-off procedure a large

\footnotetext{
${ }^{4}$ This approach is useful to analyze the localization performance of networks where RFID devices have been positioned in the area without any planning. Accurate planning would be useful to minimize the number of RFID devices required to cover the entire area, to avoid coverage holes, and so forth.
}

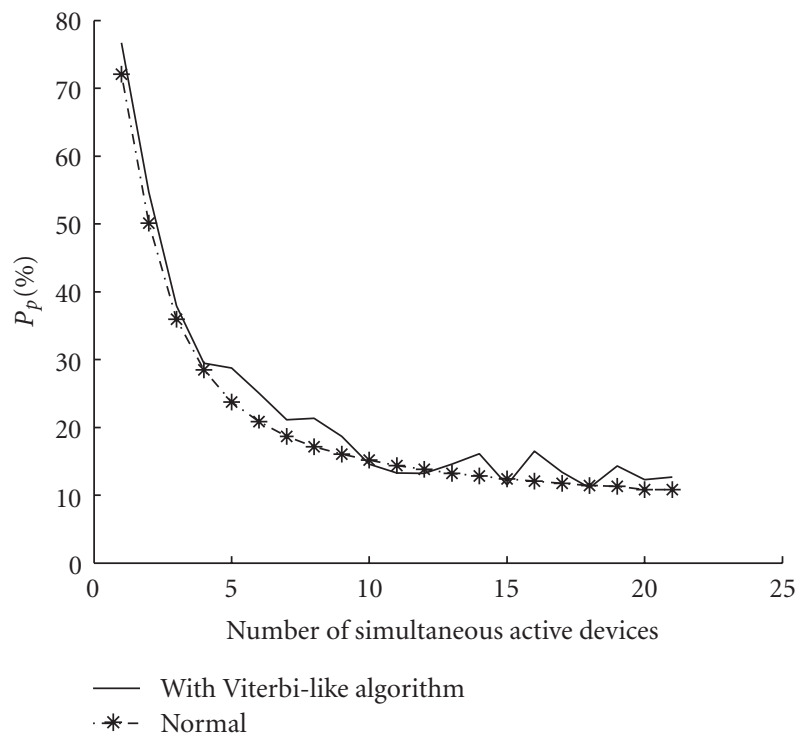

FIgURE 8: $P_{p}$ as a function of the number of active sensors in the area; terminal, Viterbi-like tracking techniques have been included.

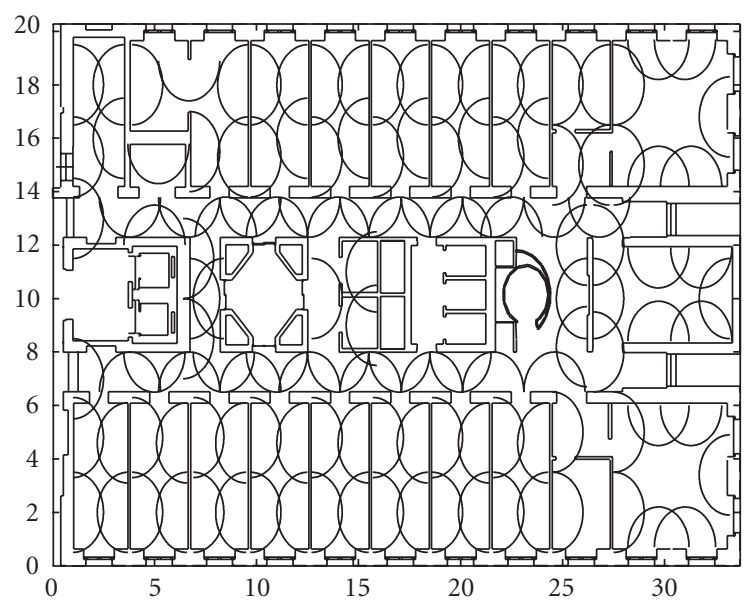

Figure 9: Layout of a subset of the passive devices in the area and illustration of their coverage area.

number of times (about 500), and for each configuration we recalculated the position of the users moving in the area. ${ }^{5}$

For simulation purposes, the coverage radius of the passive devices is restricted to $1.5 \mathrm{~m}$. To this aim, we assumed that the power of the interrogation signal is $w_{T}=20 \mathrm{dBm}$ and the sensitivity of the terminal receiver was set to $-90 \mathrm{dBm}, G_{t x}=G_{r x}=0 \mathrm{~dB}$ (omnidirectional antennas) and insertion loss $I_{L}=20 \mathrm{~dB}$. When the maximum value of $d_{i}$ is below $1.5 \mathrm{~m}$, the free-space propagation model applies. The terminal to be located was randomly positioned in the area

\footnotetext{
${ }^{5}$ As a final remark, it should be observed that the considered statistical approach allows to account for graceful performance degradation due to RFID density reduction caused by (possible) random failures of the RFID devices in the network.
} 


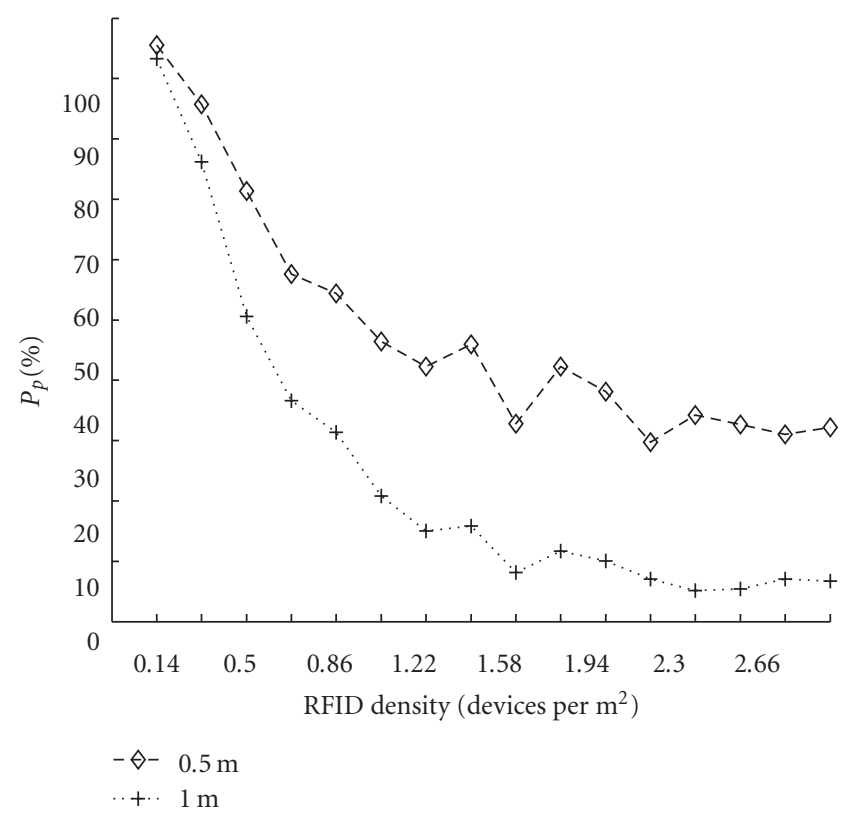

Figure 10: $P_{p}$ as a function of the density of the passive devices.

in accordance to a uniform spatial distribution. Finally, the power measured by the terminal receiver for each responding device was affected by a zero-mean Gaussian random noise with standard deviation $\sigma=2.5 \mathrm{dBm}$. Quantization of the measured power was also considered in the simulation.

In Figure 10 we plot the average $P_{p}$ as a function of the density of passive devices. Two different values for the tolerable estimation error, $0.5 \mathrm{~m}$ and $1 \mathrm{~m}$, have been considered. The average of $P_{p}$ is obtained with respect to the positions of the terminal to be located. As expected, the $P_{p}$ decreases with the density of devices. The largest values of $P_{p}$ are obtained when the number of responding passive devices is 0 or 1 . In the first case ( 0 passive device responding), position calculation cannot be performed. In the second case (1 passive device responding), the terminal can be located on a circle at distance $d_{i}$ from the passive device. In both cases, we assume that position cannot be correctly estimated and a localization error always occurs. When the number of responding devices is 2, two points represent the solution of the nonlinear system of equations in (7). In this case, the terminal position is randomly selected with equal probability between the two available.

In Figure 11 we plot the probability that the number of answering devices is equal to 0,1 or 2 or 3 or above 3 as a function of the density of the passive devices. As expected, the percentages of having 0 or 1 answering device decreases with the density and so does $P_{p}$.

Introducing the position error as the distance between the estimated point and the actual position of the terminal in the area, in Figure 12 we plot the average position estimation error as a function of the density of the passive devices. Data corresponding to 0 and 1 responding devices have not been included in Figure 12. In general, it can be observed

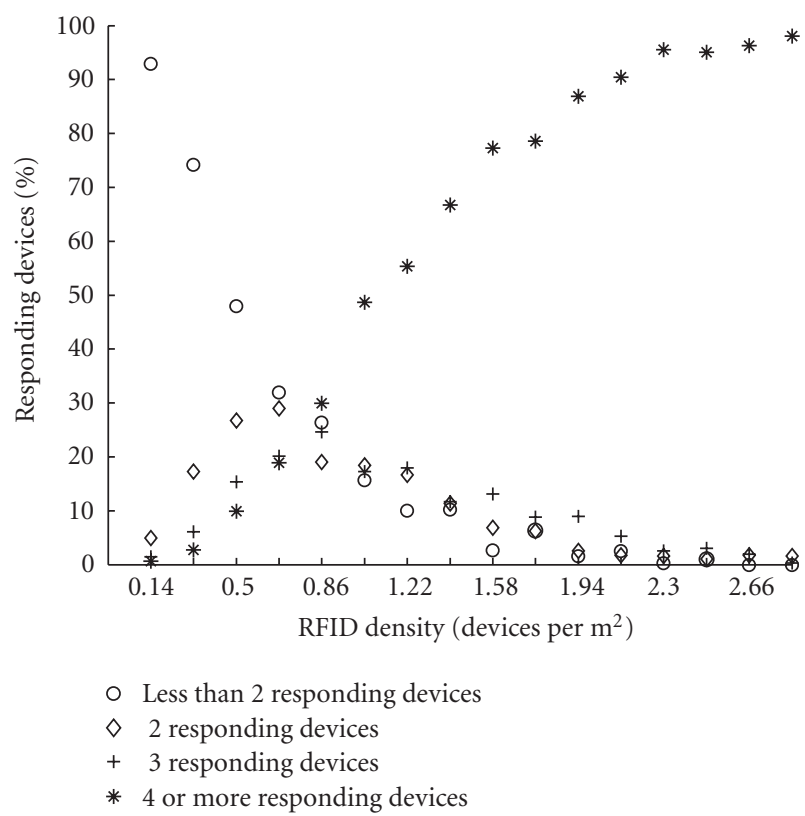

FIgURE 11: Number of passive devices responding to the terminal interrogation.

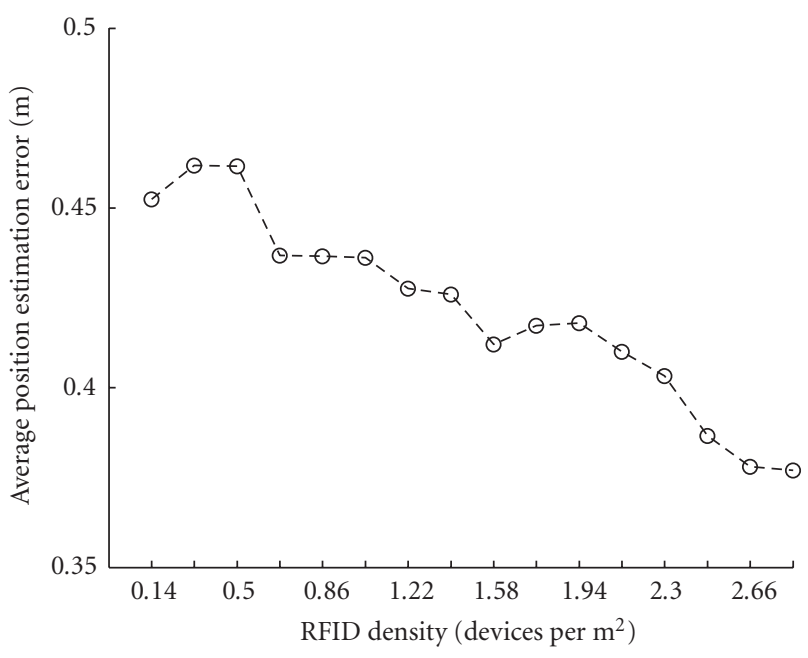

FIGURE 12: Position error as a function of the density of passive devices; power data available.

that when the number of responding devices is lower than 3 , the position error increases. This fact is shown in Figure 11 where it can be observed that for small densities, the percentage of times we have 2 responding devices is higher. From the results in Figure 12, it can be further observed that even when the number of responding devices is greater than 1 , the position estimation error remains within tolerable limits even for relatively small densities of the devices in the area. This is due to the small coverage area that allows to restrict the area where the terminal can be located. When the density of the RFID devices is sufficiently large (e.g., 2.78 devices $/ \mathrm{m}^{2}$ ), good accuracies in the position calculation can 
also be obtained using only the identities of the responding RFID devices. In this case, the server identifies the uncertainty area $U_{A}$ associated to the terminal, and in the simplest case associates the user position with one point inside $U_{A}$. In Table 2 we show the average extension of $U_{A}$ as a function of the number of RFID responding devices, $N_{\text {resp. }}$. In the same table we also indicate the average of the error between the estimated position obtained from solving (7) and the true position. When power data are not available, position error is calculated with respect to the center of $U_{A}$ that was also assumed as the estimate of the user position. As expected, in both cases, the average dimensions of the uncertainty areas decrease with the number of answering devices and accurate localization can be obtained when 3 or more RFID devices respond. The availability of uncertainty area allows to discard possible wrong solutions obtained from (7) due to noise in the measurement power and/or to possible geometrical RFID arrangements that can render the system in (7) ill-conditioned. In this case the server discards the solution obtained in (7) and defines the terminal position as the center of the uncertainty area, $U_{A}$. However, when power data can be safely used, the measurement error can be greatly reduced (see the fourth column in Table 2). ${ }^{6}$ Before concluding this section, we briefly discuss the power energy required by the interrogator to ping the RFID devices. Due to the actual market unavailability of 2, $4 \mathrm{GHz}$ RFID devices and of the corresponding interrogators, the power-energy consumption of the interrogator can be estimated assuming that the hardware used to build is based on the technology used for IEEE $802.11 \mathrm{~b}$ products. As an illustrative example, we consider the power consumptions of the Cisco Aironet PCMCIA cards indicated in [15]. In order to transmit an RF power of $100 \mathrm{~mW}$, the overall power consumption is $2.25 \mathrm{~W}$ for a transmission speed of $1 \mathrm{Mb} / \mathrm{s}$. During reception, the power consumed by the device is $1.35 \mathrm{~W}$ for receiver processing. Finally Cisco also declares a consumption of $0.075 \mathrm{~W}$ in sleep mode. Using the previous data, it is possible to obtain the average energy required to transmit one bit at $1 \mathrm{Mb} / \mathrm{s}$, that is, $E_{b}=2.25 / 10^{6}=2.25 \mu \mathrm{J} / \mathrm{bit}$. If the energy packet required to activate the RFID has an equivalent duration of 40 bits, the energy to be transmitted is $E=l \cdot E_{b}=90 \mu \mathrm{J}$. Indicating with $l$ the number of bits retransmitted by the RFID tag, the energy required in the receiver for processing is $l \cdot 1.35 / 10^{6}$. Assuming for example that $l=40$, we obtain an energy consumption of $54 \mu \mathrm{J}$ that should be added to the required transmitted energy. ${ }^{7}$ To calculate the total energy consumption required to process data obtained from tags, we need to consider the number of responding tags that can range from 1 up to 4 . In this case, the energy for the interrogation can vary from $90+54=144 \mu \mathrm{J}$ up to $90+4 \cdot 54=306 \mu \mathrm{J}$.

\footnotetext{
${ }^{6}$ During simulations, we observed that because of power measurement errors and quantization, when the number of responding devices was greater than 3, the Matlab fsolve subroutine sometimes provided unreliable results. These results were discarded in calculating the last term in column 4 of Table 2 .

${ }^{7}$ We implicitly assumed that the processing of the $l$-bits returned from the RFID should follow the same processing of a WLAN packet. This could be not true for the interrogator.
}

TABLE 2: Average extension of the uncertainty area and average distance error with and without power data.

\begin{tabular}{cccc}
\hline $\begin{array}{c}\text { Number of } \\
\text { answering } \\
\text { RFIDs }\end{array}$ & $\begin{array}{c}\text { Mean of } \\
U_{A}\left(\mathrm{~m}^{2}\right)\end{array}$ & $\begin{array}{c}\text { Average position } \\
\text { error without } \\
\text { power data }(\mathrm{m})\end{array}$ & $\begin{array}{c}\text { Average position } \\
\text { error with } \\
\text { power data }(\mathrm{m})\end{array}$ \\
\hline 1 & 3.5269 & 1.1452 & 0.6552 \\
2 & 1.5854 & 0.7423 & 0.5467 \\
3 & 0.7540 & 0.5465 & 0.2485 \\
4 & 0.4583 & 0.2933 & 0.1223 \\
\hline
\end{tabular}

Furthermore, from our simulations, the average number of responding tags in the area was 2.45 so that the average energy consumption is $90+2.45 \cdot 54=222.3 \mu \mathrm{J}$. Note that previous energy calculations assumed that RFID passive devices had a low sensitivity level, that is, they can respond even when the power at their input is very small (e.g., $-24 \mathrm{dBm}$ in our case). This corresponds to a realistic future technological objective since semiconductor techniques are rapidly advancing to reduce the RFID sensitivity towards tens of $\mu \mathrm{W}$, see [16]. If we assume $-10 \mathrm{dBm}$ [17] as a realistic value of the RFID sensitivity, applying the link budget formula in (6) for a interrogator-RFID maximum distance of $1.5 \mathrm{~m}$, we obtain a required transmitter RF power of about $2.3 \mathrm{~W}$ (in line with the data in the current literature [18]) which corresponds to an overall power consumption of about $11.5 \mathrm{~W}$.

Previous energy calculations can be used in the planning of the RFID network in order to set the polling frequency of interrogation in order to optimize the battery duration. Polling frequency should be adaptive, that is, when the server system senses that the user remains fixed in one position for a relatively long time, polling frequency should be drastically reduced.

A final observation should concern the operating frequency of the interrogator. We assumed that interrogator operates in the same frequency band of the WLAN (e.g., $2.4 \mathrm{GHz}$ ) which is used to convey data to the central server. In this case, the WLAN packets transmitted by the terminals or by the access point can activate the RFID devices. RFID responses can create background interference noise on the received WLAN packet. This could be easily avoided if the operation frequency of the RFID devices is different from that of WLAN. Many RFID devices exist on the market having operating frequency well below the $2.4 \mathrm{GHz}$. However, the adoption of RFID devices that can be activated on the WLAN band should not be discarded a priori especially if RFID could respond to interrogation on a frequency outside the WLAN band. In this case, interrogation would be at no additional energy costs since it is generated by normal packet transmission.

\section{CONCLUSIONS}

We analyzed the performance of networks used for localization in terms of the probability of localization error. Solutions based on active and passive devices were considered. A novel and practically realizable network architecture for 
localization based on passive RFID devices has been presented. Results have been obtained by simulation considering a realistic office environment and multiwall propagation in the active device case. From the results obtained in this paper, the probability of localization error in the active case is larger than that obtained in the passive case (see Figures 5 and 10). In addition, even when active or passive devices are well positioned in the area, the probability of localization error cannot decrease below an irreducible value. This is due to noise and power measurement errors which, in the active case, greatly influence the extraction of the position information starting from the data in the RF map. The proposed solution based on passive devices seems to be preferable with respect to the active one. This is due to the possibility of increasing the density of passive devices to be used for localization at relatively low cost. The corresponding increase in the number of active devices would lead to very high costs in the active localization system in terms of maintenance (periodical change of the batteries) or installation (necessity to connect some or all the devices to a powerline). Finally, it has been observed that position estimation in the passive case can be obtained simply starting from a coarse estimation based only on the uncertainty area and can be possibly refined using the measure of the powers received by the responding RFID devices. When the number of responding devices is relatively large, the accuracy of the coarse estimation is acceptable as it is also shown in Table 2.

\section{ACKNOWLEDGMENTS}

The authors would like to thank the anonymous reviewers for careful review and for valuable comments and suggestions that have been useful to improve the presentation of the paper. This work has been done within PULSERS Phase II - IST Contract N. 27142 of the FP6 of the European Community.

\section{REFERENCES}

[1] J. Hightower and G. Borriello, "A survey and taxonomy of location systems for ubiquitous computing," Technical Report UW-CSE 01-08-03, August 2001.

[2] P. Bahl, V. N. Padmanabhan, and A. Balachandran, "A software system for locating mobile users: design, evaluation, and lessons," MSR Technical Report MSR-TR-2000-12, Microsoft Research and University of California, San Diego, Calif, USA, February 2000.

[3] F. Van Diggelen and C. Abraham, "Indoor GPS Technology," CTIA, Dallas, Tex, USA, May 2001.

[4] P. Prasithsangareel, P. Krishnamurty, and P. K. Chrysantis, "On indoor position location with wireless LANS," in International Symposium on Personal, Indoors and Mobile Radio Communications (PIMRC '02), Lisboa, Portugal, September 2002.

[5] N. B. Priyantha, A. Chakraborty, and H. Baladrishnan, "The cricket location-support system," in Proceedings of the 6th Annual International Conference on Mobile Computing and Networking (MOBICOM '00), pp. 32-43, Boston, Mass, USA, August 2000 .

[6] P. Bahl and V. N. Padmanabhan, "RADAR: An in-building RFbased user location and tracking system," in Proceedings of the 19th Annual Joint Conference of the IEEE Computer and Communications Societies (INFOCOM-2000), vol. 2, pp. 775-784, Tel Aviv, Israel, March 2000.

[7] P. Bahl, N. Venkata, N. Padmanabhan, and A. Balachandran, "Enhancements to the RADAR user location and tracking systems," Microsoft Research Technical Report MSR-TR-200012, February 2000.

[8] R. J. Fontana, "Recent Applications of Ultra Wideband Radar and Communications Systems," http://www.multispectral. com.

[9] PULSERS, "Pervasive Ultra-wideband Low Spectral Energy Radio Systems," EU-IST Programme (FP6), http://www.pulsers.net.

[10] ISO/IEC 802-11: 1999(E), "Part 11: Wireless LAN Medium Access Control (MAC) and Physical Layer (PHY) Specifications" ANSI/IEEE Std 802.11, 1999 Edition.

[11] "Specification of the Bluetooth System," version: 1.205 November 2003, http://www.bluetooth.org.

[12] K. Finkenzeller, RFID Handbook: Fundamentals and Applications in Contactless Smart Cards and Identification, John Wiley \& Sons, New York, NY, USA, 2nd edition, 2003.

[13] A. Borrelli, C. Monti, M. Vari, and F. Mazzenga, "Channel models for IEEE $802.11 \mathrm{~b}$ indoor system design," in IEEE International Conference on Communications, vol. 6, pp. 37013705, Paris, France, June 2004.

[14] G. Wolfle, P. Wertz, and F. M. Landstorfer, "Performance, accuracy and generalization capability of indoor propagation models in different types of buildings," in Proceedings of 10th IEEE International Symposium on Personal, Indoor and Mobile Radio Communications (PIMRC '99), Osaka, Japan, September 1999 .

[15] http://www.cisco.com.

[16] http://www.trolleyscan.com/paper/ecolect.html.

[17] Philips EPC 1.19 G2 RFID ASIC, http://www.semiconductors. philips.com.

[18] http://www.alientechnology.com.

Damiano De Luca received the "Laurea" degree in telecommunication engineering from the University of Rome "Tor Vergata," Rome, Italy, in 2004. After his degree, in 2004, he joined RadioLabs, consortium between the University of "Tor Vergata" in Rome and Italian Industries operating in the field of wireless communications. His research interests include UWB, Bluetooth, and wireless lan technologies. He

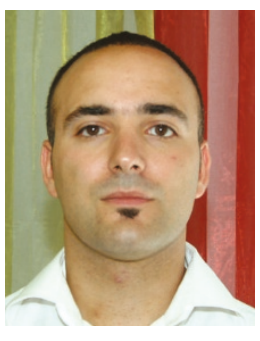
is involved the radio propagation in indoor environment analysis, based on both empirical models (free-space modified, MotleyKeenan model, multiwall model) for the characterization of the radio coverage power and deterministic models (ray tracing and ray launching) for the theoretical characterization of channel models.

Franco Mazzenga received the Dr. Ing. degree in electronic engineering cum laude from the University of Rome "Tor Vergata," Italy in 1993. From 1993 to 1994, he was with Fondazione Ugo Bordoni making research on the propagation at millimeter waves. In 1997, he obtained the Ph.D. degree in telecommunications. From 1998 up to 2000, he was with the Consorzio di Ricerca in Telecommunicazioni (CoRiTel).

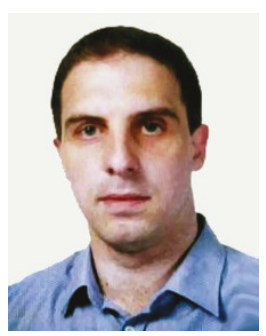


Presently he is a Researcher in the Electronic Engineering Department of the University of Rome "Tor Vergata," Italy. He is the author of several scientific publications and the coauthor of a book on radar systems (in Italian). His main interests are in statistical signal processing, estimation theory, $3 \mathrm{G}$ and $4 \mathrm{G}$ networks. He is the Technical Director of the RadioLabs Consortium (www.radiolabs.it).

Cristiano Monti received the "Laurea" degree in electronic engineering from the University of Rome "Tor Vergata" in 2000. He also received an M.S. degree in business administration in 2002. In the university, he was involved on IEEE 802.11 technology research. In particular his main activity was on 2,4 , and $5 \mathrm{GHz}$ channel modellings and network management (load balancing, security, and handoff issues among different

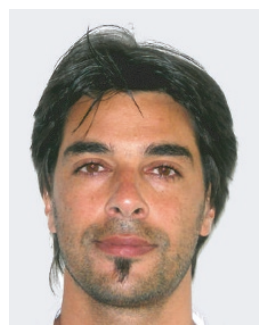
networks). Now he is working to receive the Ph.D. degree in University of Rome "Tor Vergata." He just takes care to research about indoor localization using sensor networks, RFID, UWB. Furthermore, he is studying the problem about WiMax coverage and interference.

Marco Vari received the Telecommunications Engineering degree in telecommunications engineering at the University of Rome "Tor Vergata" in 2002. His thesis focused on coexistence between WLAN (IEEE $802.11 \mathrm{~b}$ ) and Bluetooth devices. In 2003, he developed in the Tor Vergata Campus a WiFi network to provide free internet connectivity to students. His interests are in wireless network and in indoor localization

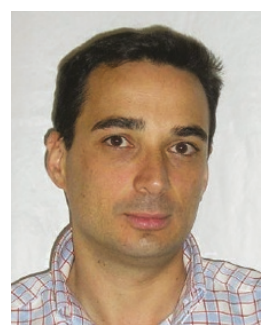
techniques. Now he is studying an indoor localization technique based on an autolearning system. 